Design, Modelling and Fabrication of Advanced Robots

Vol: 1(1), 2022

REST Publisher; ISBN: 978-81-948459-3-5

Website: http://restpublisher.com/book-series/design-modelling-and-fabrication-ofadvanced-robots/

\title{
Development of iot Based Water Quality Control and Filtration System
}

\author{
G. Shanmugasundar, G.Manoj Kumar, S. E. Gouthem, V. Surya Prakash, R. Vijai Karthik \\ Associate Professor Mechanical Engineering, Sri Sai Ram Institute of Technology, Chennai, Tamilnadu, India. \\ Department of Mechanical Engineering, Sri Sai Ram Institute of Technology, Chennai, Tamilnadu, India. \\ *Corresponding author Email:shanmugasundar.mech@sairamit.edu.in
}

\begin{abstract}
Water is the basic and essential resource which is required for every need such as agriculture, industrial activity and for domestic purpose. Especially in the industrial areas although many water monitoring systems were planted, the waste water disposal is minimally controlled. This project not only helps to monitor water at the industrial level but it can be used anywhere water quality plays a major role. The water quality is continuously monitored with the help of sensors (PH, Temperature, Turbidity, conductivity, water level indicator). It also indicates the level of the water and checking the quality of the water with the help of the valve control system, it sends the water with impurities for the filtration system. The entire system is completely controlled by IOT system and it is continuously monitored by the module.
\end{abstract}

\section{Introduction}

According to the statistical report produced by Central Pollution Control Board (CPCB) in the year 2021, it shows that out of 1400 rivers 1351 rivers are polluted. It also states that the major reason for the pollution of rivers is due to the agricultural activity which contributes a major ratio for the contamination of the water bodies and then the various processes performed in the industrial areas where the disposal of the effluents and other wastes are discharged into the water bodies also pollutes the water and domestic activities also shares a small amount for the contamination of the water. Although several measures and precautions were taken for minimizing the rate of pollution of the water bodies it is not much effective and showed impact for the cause. The pollutants due to the agricultural activities are the pesticides and fertilizers which are getting washed away from the fields and reach the river water. These pollutants are requiring proper filtration and separation technique and this system constantly checks the impurities through IOT module and this helps to provide complete filtration to the level where water is fit for regular usage. It is also much useful for treating the water from industrial plants. The process is more simple and efficient in a cost effective manner.

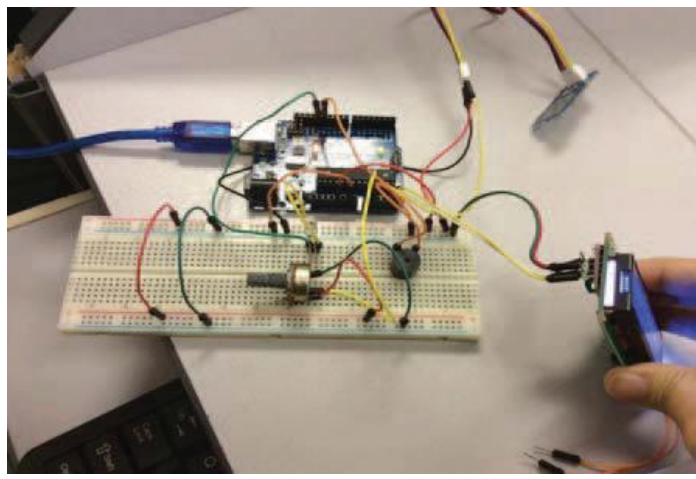

FIGURE 1. Iot Based Water Management System

\section{Sensors}

$\mathrm{PH}$ sensor is the representation of the concentration of the hydrogen ion per liter in the solution. It varies between 0 to 14 . It can be also termed as the logarithmic concentration of moles in the hydrogen ions per liter of solution. The solution having $\mathrm{PH}$ value of 0 to 7 is acidic solution with large concentration of hydrogen ions whereas if the PH value is between 8 to 14 , then it is basic solution with less concentration ion in the solution. A PH sensor has measuring and a reference electrode. A battery positive terminal is connected to the measuring electrode and the negative terminal is connected to the reference electrode. Due the difference in conductivity of the measured $\mathrm{PH}$ value is indicated through this difference of the sensor.Conductivity sensorSalts get discharged into positive and negative ions. The dissipated ions are the conductors and the conductivity is the ability of the water to conduct electricity. The positively charged ions are sodium, potassium, 
magnesium and calcium and the negatively charged ion are carbonate, bicarbonate, chloride, nitrate, phosphate. Electrical conductivity is measured with the help of the probe and the meter.
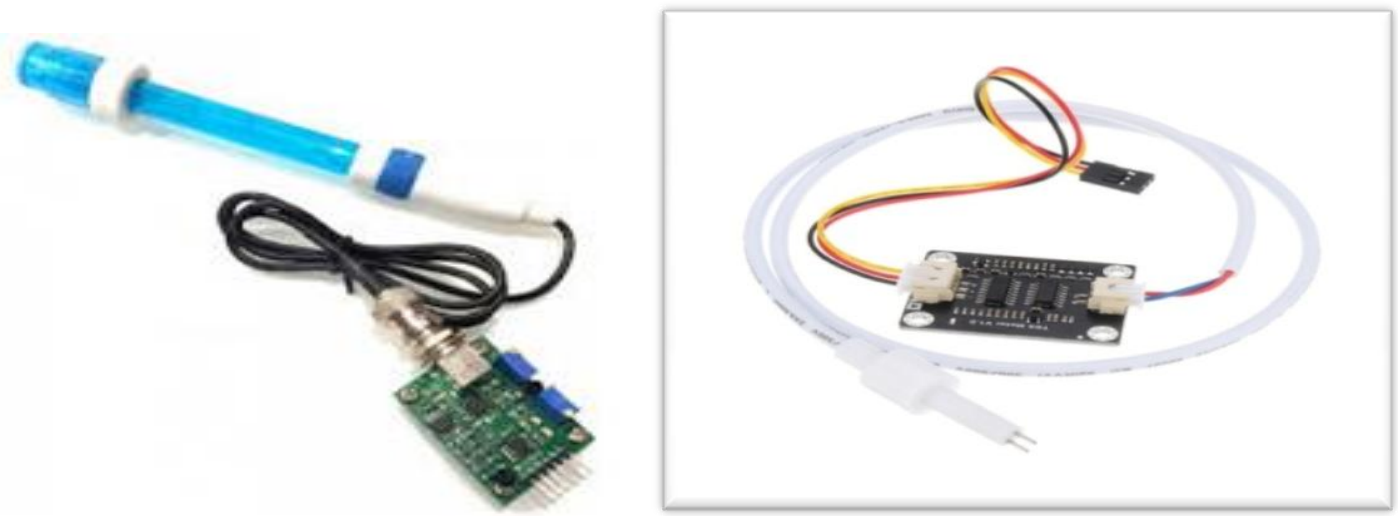

FIGURE 2. PH sensor moduleFIGURE 3. conductivity sensor

The probes consist of two metal strips separated by $1 \mathrm{~cm}$ apart from each other and they are measured by mili or microSiemens per $\mathrm{cm}$. The amount of current flowing through the water is directly proportional to the amount of discharged ions in the solution which is measured through conductivity. Temperature sensorThe basic principle of the temperature sensor is difference in voltage between the diode terminals. This type of the sensor uses solid state technique to estimate the temperature of the sensor. If the temperature increases the voltage difference is also indicated. They also use bimetallic strips to indicate the temperature level in the sensor. Water Level IndicatorWater level indicator works by using the sensor probe to indicate the water level in the storage tank. These probes send information to the control panel to trigger an alarm or indicator. Water level indicator uses a 2-way system, control panel and a probe sensor. Depending on the configuration of the system it can be used maximum up to 6 sensor probes.
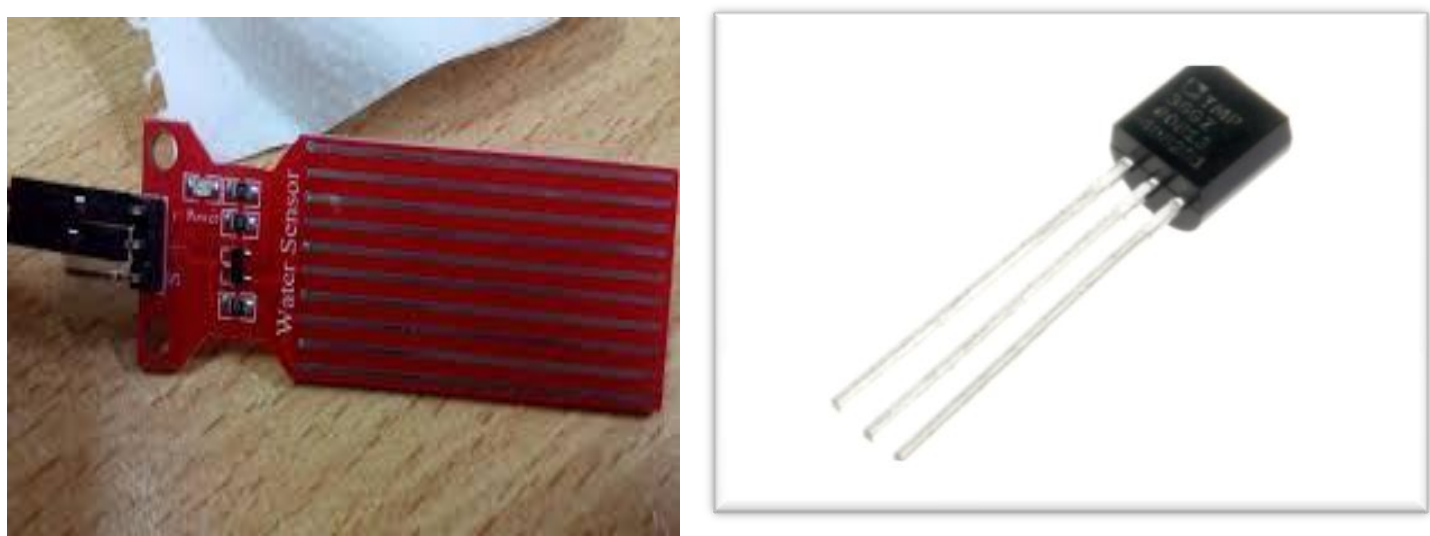

FIGURE 4. Water Level Indicator Module FIGURE 5.Temperature Sensor Module

Turbidity sensorTurbidity is the measure of the amount of the impurities suspended particles in the fluid. It is the soil in the water or impurities in the water which estimate the impurities in the fluid. There are several industries and other places where the uses this water and by using this sensor we can estimate the impurities. This senor works on the principle by amount of light transmitted through the sample is dependent on the amount of soil in the water. The turbidity sensor measures the amount of light water in clean water and the turbidity in water is relative both the samples. In this project this sensor indicates the quality of the project and with the help of this sensor the quality of the water is indicated at instant. Filtration system The filtration system is completely based on the output from the PH sensor which gives the state of purity of the water. Based on this output, the water is transported to the filtration unit where the water gets purified and sends to the outlet. This type of the system varies depend upon the capacity of the water and for what purpose it is installed for.Mechanical filtration removes particles in two ways; through surface and depth filtration. Many particles are removed by surface filtration, a sieving process that traps large particles on the top or leading surface of the filter. Smaller particles pass through the surface layer and are subsequently removed through depth filtration. With depth filtration, smaller particles become trapped as they make their way through the increasingly smaller pores in a filter. It can have done with the help of process like reverse osmosis, coagulation filtration etc. 


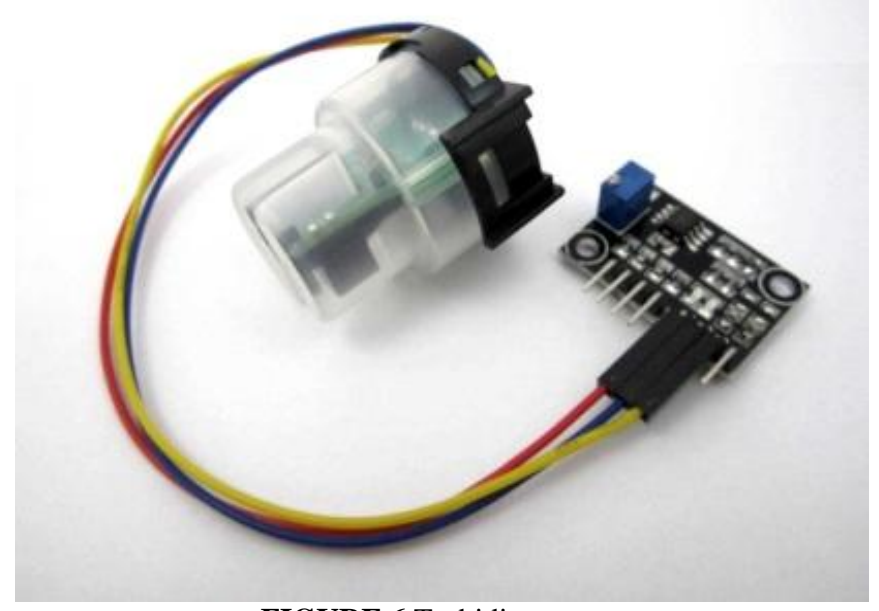

FIGURE 6.Turbidity sensor

\section{Flowchart/Work flow diagram}

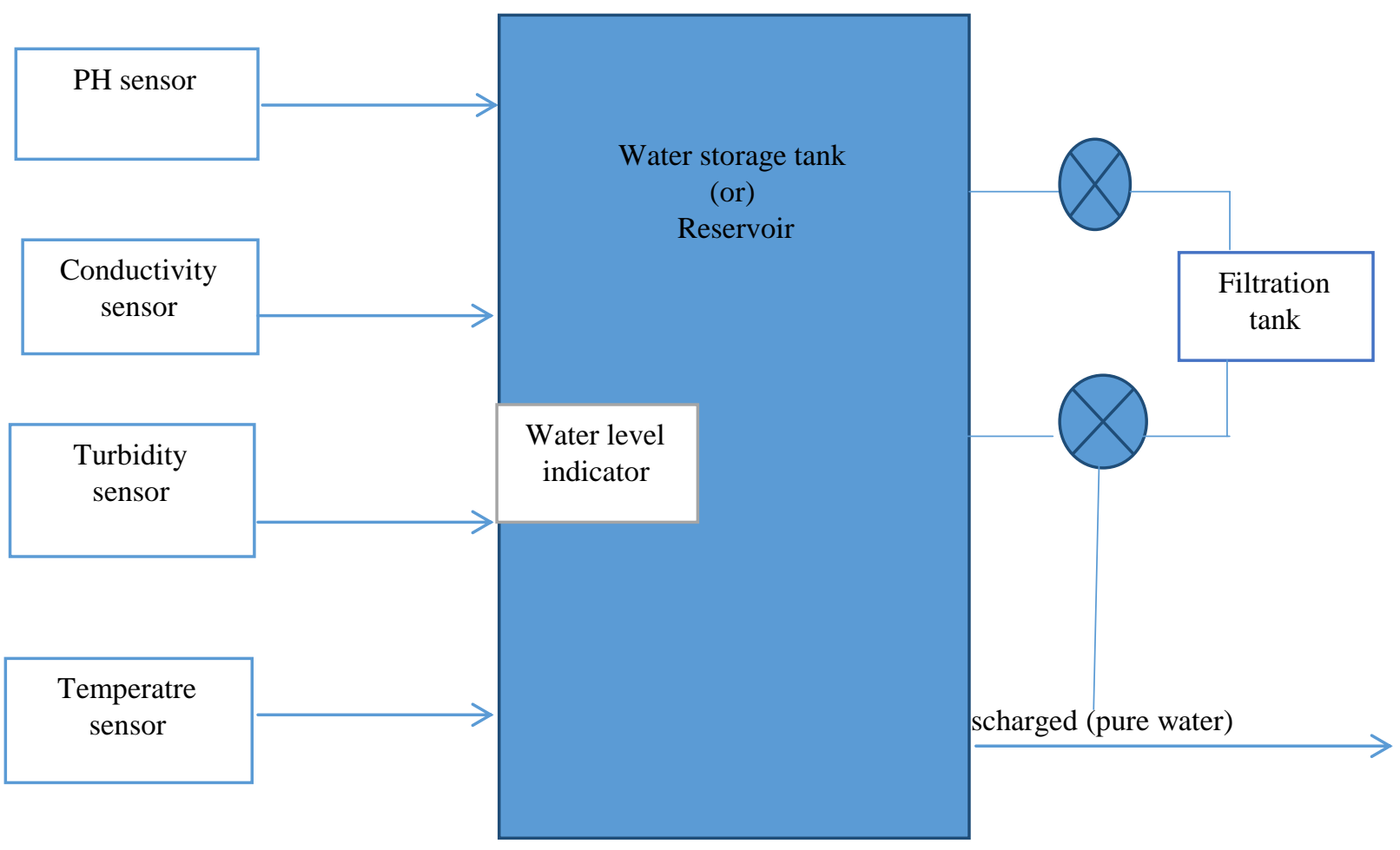

FIGURE 7. Flowchart /Work flow diagram

\section{Prototype Implementation}

The control of all the sensors is given to the arduino controller where the sensing unit of the sensor is immersed into the tank of the water. ${ }^{[3]}$ The four sensors ( $\mathrm{PH}$, temperature, conductivity, turbidity) continuously capture the data's from the module in the analog signals and converts it into digital signal by the arduino. All the sensors are interconnected through the microcontroller and any change in the sensor can be made with the help of this system. The sensors are interconnected with the help of the Bluetooth or WIFI module. The PH and the turbidity sensor indicates the quality of the water and the output will be given to the valve control system where if it finds the water needs to be treated it will send the water for the filtration otherwise it will send the water for discharge. In the filtration system depending upon the capacity of the water the filtration system can be chosen. After the filtration of the water it is again send for checking the quality for complete removal of the impurity and then it is purified it is discharged else the process repeats until the water gets purified. ${ }^{[2]}$ All the sensors are 
IOT controlled (Zigbee module for short ranges, WIFI for long range of system) which helps to see the output of each sensors in the mobile or PC system and the control of the sensors can be done with this module.

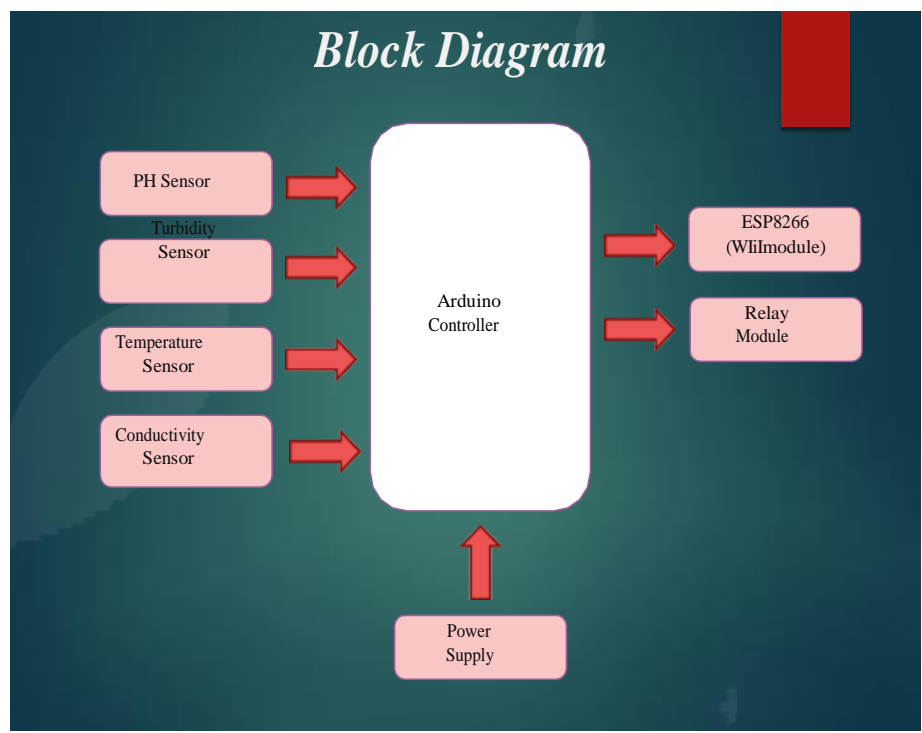

FIGURE 8. Block diagram of working of the system.

\section{Conclusion}

IOT is becoming part of our technology where control can have made easier the access of the system is more convenient. This prototype uses IOT module in order to monitor the quality of the water continuously in order to minimize and eradicate the contamination of the water due to the industrial and other activities. Although many improvements were made in the system to reduce the pollution automatic control over monitoring the system for the discharge of the water provides better output when compared to the manual checking of the system. This also gives a long range life cycle of the system with a minimum maintenance required to be given and the resultant cost of the system is also very low. This system of monitoring also gives a way far less pollution of water and it can be reduced and can also be reused.

\section{References}

1. Water pollution Statistical report given by CPCB, https://cpcb.nic.in/nwmp-data-2019/

2. IOT and arduino control of the module is given with the help of this site, https://create.arduino.cc/projecthub/ahmedibrrahim/iot-using-esp8266-01-and-arduino-afa35e

3. Tuan Le Din, design and development of robust based senor technology.

4. Overview of Ganga river basin report by central pollution control board, http://www.cpcbenvis.nic.in/cpcb_newsletter/bio\%20mapping\%20of\%20river.pdf

5. PH sensor reference www.omega.co.uk/prodinfo/pH-meter

6. Shanmugasundar, G., Dharanidharan, M., Vishwa, D., \& Sanjeev Kumar, A. P. (2021). Design, analysis and topology optimization of connecting rod. Materials Today: Proceedings, 46, 3430-3438.

7. G Shanmugasundar, M Dharanidharan, D Vishwa, A Jayaprakash and P Abimanyu , 2020, "Design and Finite Element Analysis of Prosthetic Hand Controlled by Wireless Gestures for Differently-abled People" IOP Conference Series: Materials Science and Engineering 923 (2020) 012019 IOP Publishing, Doi:10.1088/1757899X/923/1/012019.

8. G. Shanmugasundar, G. Fenneth Moses, S. Jayachandran, V.D. Rathnavel Subramanian and R. Rajagopalan, 2020 "Design and Fabrication of Solar Powered Multi-Purpose Agricultural Vehicle with IOT Control" , Journal of Advanced Research in Dynamical and Control Systems, Volume 12, Pages: 1928-1933.

9. G.Shanmugasundar, R.Yokesh, S.Yuvaranjith, R.Barath, S.Balasubramanian (2020), Design and Fabrication of Intelligent Gas stove for women safety , International journal of pharmaceutical research , https://doi.org/10.31838/ijpr/2020.12.02.0126

10. G.Shanmugasundar, M.Dharanidharan,, D.Vishwa, P. Gokul, (2020), Design, Modelling and Analysis of an Autonomous Ornithopter Robot for Environmental Pollution Monitoring, Interciencia Journal (ISSN: 0378-1844), 45(12 ), PP- 2-10. https://intercienciajournal.com/intercien/index.php/pdf/stream/54un4/1604942593.

11. G. Shanmugasundar, P. Jagadeeshwar, S. Adithya, V. Nagappan, and M. Bhaskar, "Design, fabrication and analysis of personal vacuum assisted climber," in Journal of Physics: Conference Series, 2019, vol. 1362, no. 1, doi: $10.1088 / 1742-6596 / 1362 / 1 / 012057$.

12. G. Shanmugasundar, R. Sivaramakrishnan, S. Meganathan, and S. Balasubramani, "Structural optimization of an five degrees of freedom (T-3R-T) robot manipultor using finite element analysis," in Materials Today: Proceedings, 
2019, vol. 16, pp. 1325-1332, doi: 10.1016/j.matpr.2019.05.231.

13. G. Shanmugasundar, R. Sivaramakrishnan, and S. Balasubramani, "Method of Trajectory Generation of a Generic Robot using Bresenham's Circle Algorithm," Indian J. Sci. Technol., vol. 9, no. 48, 2017, doi: 10.17485/ijst/2016/v9i48/108476.

14. R. Sridhar, G. Shanmugasundar, and A. Srithar, "A Geometrical Modular Design for Handling of LPG Cylinders using Nested Kinematic Robotic Gripper," in Indian Journal of Science and Technology, 2016, vol. 9, no. 48, doi: 10.17485/ijst/2016/v9i48/108474.

15. Godbole, Nishant, Shajit Yadav, M. Ramachandran, and Sateesh Belemkar. "A review on surface treatment of stainless steel orthopedic implants." Int J Pharm Sci Rev Res 36, no. 1 (2016): 190-4.

16. G. Shanmugasundar and R. Sivaramakrishnan, "Design and analysis of a newly developed seven degree of freedom robot for inspection,” Int. J. Control Theory Appl., vol. 9, no. 24, pp. 393-402, 2016.

17. G. Shanmugasundar and R. Sivaramakrishnan, "Computer aided modelling and simulation of a generic robot for inspection,” Int. J. Appl. Eng. Res., vol. 10, no. 24, pp. 44049-44056, 2015.

18. G. Shanmugasundar, R. Sivaramakrishnan, R. Sridhar, and M. Rajmohan, "Computer Aided Modelling and Static Analysis of an Inspection Robot," in Applied Mechanics and Materials, 2015, vol. 766-767, doi: 10.4028/www.scientific.net/amm.766-767.1055.

19. G. Shanmugasundar, R. Sivaramakrishnan, and M. Rajmohan, "Computer aided simulation for workspace plot of a newly designed inspection robot," in 2014 IEEE International Conference on Computational Intelligence and Computing Research, IEEE ICCIC 2014, 2015, doi: 10.1109/ICCIC.2014.7238470. 